\title{
Electrical Stimulation of the Vagus Nerve Dermatome in the External Ear is Protective in Rat Cerebral Ischemia
}

\section{Citation}

Ay, Ilknur, Vitaly Napadow, and Hakan Ay. 2015. "Electrical Stimulation of the Vagus Nerve Dermatome in the External Ear Is Protective in Rat Cerebral Ischemia." Brain Stimulation 8 (1) (January): 7-12. doi:10.1016/j.brs.2014.09.009.

\section{Published Version}

10.1016/j.brs.2014.09.009

\section{Permanent link}

http://nrs.harvard.edu/urn-3:HUL.InstRepos:37047484

\section{Terms of Use}

This article was downloaded from Harvard University's DASH repository, and is made available under the terms and conditions applicable to Open Access Policy Articles, as set forth at http:// nrs.harvard.edu/urn-3:HUL.InstRepos:dash.current.terms-of-use\#OAP

\section{Share Your Story}

The Harvard community has made this article openly available.

Please share how this access benefits you. Submit a story.

\section{Accessibility}




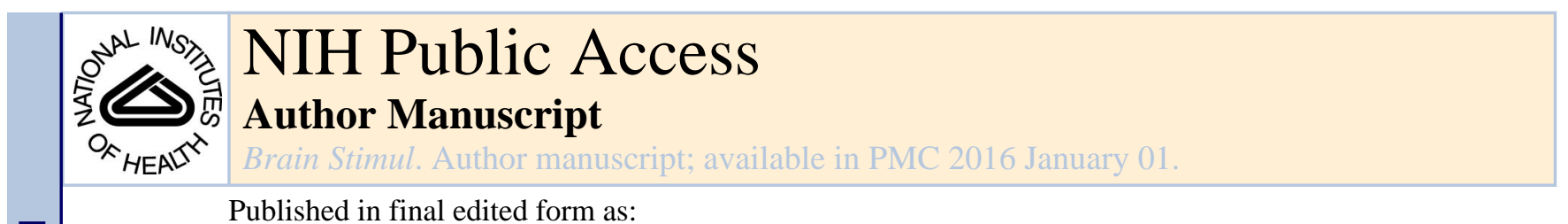

Published in final edited form as:

Brain Stimul. 2015 ; 8(1): 7-12. doi:10.1016/j.brs.2014.09.009.

\title{
ELECTRICAL STIMULATION OF THE VAGUS NERVE DERMATOME IN THE EXTERNAL EAR IS PROTECTIVE IN RAT CEREBRAL ISCHEMIA
}

\author{
Ilknur Ay, MD, PhDa, ${ }^{a}$, Vitaly Napadow, PhD ${ }^{a, b}$, and Hakan Ay, MD ${ }^{a}$ \\ aMGH/MIT/HMS Athinoula A. Martinos Center for Biomedical Imaging, Massachusetts General \\ Hospital East, $14913^{\text {th }}$ Street, Charlestown, MA, 02129, USA \\ bDepartment of Biomedical Engineering, Kyunghee University, Yongin, Korea
}

\begin{abstract}
Background-Although cervical vagus nerve stimulation is effective for reducing infarct volume in rats, it is not feasible for acute human stroke as it requires surgical incision of the neck. We hypothesized that stimulation of the dermatome in the external ear innervated by the vagus nerve (auricular vagus nerve stimulation; aVNS) reduces infarct volume after transient focal ischemia in rats.
\end{abstract}

Methods-Animals were randomized to active aVNS or sham stimulation. For aVNS, electrical stimulation of the left cavum concha (1 hour duration) using percutaneous needles was initiated 30 min after induction of ischemia. Behavioral and tissue outcome were measured 24 hours after induction of ischemia. In a separate experimental dataset, c-Fos immunohistochemistry was performed to identify the brain regions activated after the stimulation.

Results-Stimulation of the left cavum concha resulted in bilateral c-Fos staining in the nuclei tractus solitarii and the loci coerulei in all animals. There was no c-Fos staining in any part of the brainstem in sham control animals. The mean infarct volume (SD) as calculated by indirect method was $44.20 \pm 7.58 \%$ in controls and $31.65 \pm 9.67 \%$ in treated animals $(\mathrm{p}<0.0001)$. The effect of aVNS on tissue outcome was associated with better neurological scores at 24 hours after ischemia $(\mathrm{p}<0.0001)$.

Conclusions-Electric stimulation of the vagus nerve dermatome in the external ear activates brainstem afferent vagal nuclei and reduces infarct volume in rats. This finding has potential to facilitate the development of treatments that leverage the brain's endogenous neuroprotective pathways at the setting of acute ischemic stroke.

(C) 2014 Elsevier Inc. All rights reserved.

*Corresponding author: Ilknur Ay, MD, PhD, Athinoula A. Martinos Center for Biomedical Imaging, Massachusetts General Hospital East, $14913^{\text {th }}$ Street, Suite 2301, Charlestown, MA, 02129, USA, Phone: 1-617-724-1145; Fax: 1-617-726-7422, iay@mgh.harvard.edu.

Disclosures: None

Publisher's Disclaimer: This is a PDF file of an unedited manuscript that has been accepted for publication. As a service to our customers we are providing this early version of the manuscript. The manuscript will undergo copyediting, typesetting, and review of the resulting proof before it is published in its final citable form. Please note that during the production process errors may be discovered which could affect the content, and all legal disclaimers that apply to the journal pertain. 


\section{Keywords}

Cerebral ischemia; vagus nerve stimulation; Arnold's nerve; neuroprotection; c-Fos; rat

\section{Introduction}

Cervical vagus nerve stimulation (cVNS), initiated $30 \mathrm{~min}$ after middle cerebral artery occlusion (MCAO), reduces infarct volume by up to $50 \%$ in rats $[1,2]$. The effect of cVNS on tissue outcome is associated with significant and persistent improvement in functional outcome [3]. Although cVNS is effective in experimental models of cerebral ischemia, the procedure itself is not feasible in the setting of acute human stroke as it requires surgical incision of the neck and implantation of stimulation electrodes and a pulse generator. A simple and non-invasive means of stimulation is needed to develop vagus nerve stimulation as a viable treatment for acute human stroke.

While the vagus nerve is primarily a viscero-motor and viscero-sensory nerve, it has also somatic-sensory function where it conducts sensation from the larynx, pharynx, external auditory canal, external surface of the tympanic membrane, and the meninges of the posterior cranial fossa [4]. It has been suggested that stimulation of the dermatome corresponding to the auricular branch of the vagus nerve (Arnold's nerve) in the external ear may be a non-invasive alternative to cVNS (Fig. 1) [5-8]. This is the region where the familiar "ear-cough reflex" can be elicited upon cutaneous stimulation and the dermatome is quite similar between rats and humans $[9,10]$. Stimulation of this region in the ear provides seizure control in animals and induces antinociceptive effects, similar to cVNS, in humans $[9,11,12]$. In the present study, we sought to determine whether auricular vagus nerve stimulation (aVNS) activated the same vagal nuclei activated by the gold standard cVNS and whether this was associated with a reduction in infarct volume and improvement in functional outcomes in a model of MCAO in rats.

\section{Material and methods}

\section{1- The effect of aVNS on infarct size}

Thirty four adult male Wistar rats (350-400 g, 10-13 weeks of age, Charles River Laboratories, Wilmington, MA) were used. All studies were conducted in accordance with the NIH Guide for the Care and Use of Laboratory Animals and the regulations of the Massachusetts General Hospital. Physiological monitoring, ischemia surgery, and neurological assessment were performed as previously reported [2]. In brief, animals were anesthetized by isoflurane (4-5\% for induction, $1-2 \%$ for maintenance in $30 \%$ oxygen and $70 \%$ nitrous oxide) and rectal temperature was maintained at $37^{\circ} \mathrm{C}$ to $37.5^{\circ} \mathrm{C}$ using a homeothermic pad. As an indirect measure of brain temperature, the left temporalis muscle temperature was measured continuously throughout the study using a T-type implantable thermocouple probe and kept constant at $37^{\circ} \mathrm{C}$ to $37.5^{\circ} \mathrm{C}$ using an incandescent lamp. A PE-50 cathether was inserted into the right femoral artery to monitor arterial blood pressure (ABP), heart rate (HR), blood gases, and $\mathrm{pH}$. A burr hole was drilled $5 \mathrm{~mm}$ right lateral and $1 \mathrm{~mm}$ posterior to bregma and the laser Doppler flowmeter probe was placed above the dura 
to continuously record regional cerebral blood flow (rCBF) starting from prior to the onset of ischemia until early after reperfusion (Blood Flow Meter, ADInstruments, Colorado Springs, CO) [13-15]. Transient right MCAO was induced by an intraarterial filament (diameter: $0.39 \pm 0.02 \mathrm{~mm}$; Doccol Corporation, Redlands, CA) and lasted for 2 hours $[1,16]$.

Animals were randomly assigned to aVNS treatment $(\mathrm{n}=15)$ or control group $(\mathrm{n}=16)$ by computer generated algorithm. aVNS was performed using two acupuncture needles (38 gauge, stainless steel with silver handle; Cloud Dragon, China) connected to a Grass Model S48 stimulator and constant current unit (Grass Instruments, West Warwick, RI). The needles were inserted $0.5-1 \mathrm{~mm}$ under the skin over the left cavum concha $15 \mathrm{~min}$ after MCAO (Fig 1). A $30 \mathrm{sec}$ train of stimulation consisting of $0.5 \mathrm{msec}$ square pulses ( $0.5 \mathrm{~mA}$ ) delivered at $20 \mathrm{~Hz}$ was initiated $30 \mathrm{~min}$ after MCAO. Stimulation was repeated at every 5 min for one hour [1,17]. All the procedures including electrode implantation were replicated in the control animals except delivery of electrical stimulation.

At the end of the ischemic period, the intraarterial filament was removed and reperfusion was instituted. All catheters and electrodes were removed, and incisions were sutured 20 min after reperfusion. Bupivacaine $(0.25 \%$; topical $)$ and buprenorphine $\mathrm{HCl}(0.05 \mathrm{mg} / \mathrm{kg}$; sc) was given to ameliorate surgical pain, and animals were allowed to awaken from anesthesia. Functional outcome was assessed using a five-point scale that measures forelimb flexion, resistance, and circling behavior at 3 hours and 24 hours after ischemia $[16,18]$.

Animals were euthanized by $\mathrm{CO}_{2}$ inhalation 24 hours after ischemia. The brain was removed and the cerebrum was sliced into seven $2 \mathrm{~mm}$-thick coronal sections. After incubation with 2,3,5-triphenyltetrazolium chloride (TTC; 2\%, $30 \mathrm{~min}$ at room temperature) followed by $10 \%$ formalin ( 48 hours at $4{ }^{\circ} \mathrm{C}$ ), digital images of the sections were acquired. Areas of interest (infarct area, ipsilateral non-infarct area, and contralateral hemispheric area) were manually outlined in a blinded fashion, using Image $\mathbf{J}(\mathrm{NIH})$. Infarct volume of each brain was determined using both direct and indirect methods. For this, the infarct area (direct method) or the corrected infarct area (contralateral hemispheric area minus ipsilateral non-infarct area; indirect method) was multiplied by slice thickness and the volume was expressed as a percentage of the contralateral hemispheric volume.

\section{2- Brain regions activated by aVNS}

We performed c-Fos immunohistochemistry in order to identify the brain regions activated after the stimulation in a separate set of animals $(n=12)$. We used the same ischemia stimulation protocol outlined before. There were 4 experimental groups: 1) aVNS group $(\mathrm{n}=3)$ : 1 hour electrical stimulation of the cavum concha was performed, 2) control group $(\mathrm{n}=3)$ : electrodes were implanted into the cavum concha but no stimulus was delivered, 3 ) $c V N S$ group $(\mathrm{n}=3)$ : self-constructed stimulation electrodes were implanted into the right cervical vagus nerve and 1 hour electrical stimulation was performed (positive control), 4) trigeminal nerve stimulation group $(\mathrm{n}=3)$ : the acupuncture electrodes were implanted into the left lower side of the mouth and 1 hour electrical stimulation was performed (negative control). In this experiment, animals were euthanized and transcardially perfused with $4 \%$ formaldehyde in phosphate buffered saline $(\mathrm{pH}$ 7.4) 30minutes after the end of the electrical 
stimulation or 2 hours after MCAO (in contrast to 24 hours after MCAO in the prior experiment). The brains were removed and cryoprotected in $30 \%$ sucrose at $4{ }^{\circ} \mathrm{C}$ for 3 days. Using a cryostat, $30 \mu \mathrm{m}$-thick coronal sections with a $500 \mu \mathrm{m}$ interslice gap in the brainstem and cerebellum and $1500 \mu \mathrm{m}$ gap in the cerebrum were obtained. Sections were stored in cryoprotectant solution at $-20^{\circ} \mathrm{C}$. After quenching endogenous peroxidase activity with $0.3 \% \mathrm{H}_{2} \mathrm{O}_{2}$ (30 min at room temperature) and blocking with $3 \%$ normal horse serum in $0.25 \%$ Triton X-100 ( 2 hours at room temperature), free floating sections were incubated with polyclonal anti-cFos antibody (Ab-5; 1:30 000; Calbiochem, San Diego, CA) for 72 hours at $4^{\circ} \mathrm{C}$ [19]. This was followed by incubation in biotinylated horse anti-rabbit IgG (1:200; 2 hours at room temperature; Vector Laboratories, Burlingame, CA). The reaction product was amplified by Vectastain Elite ABC Kit (Vector) and visualized by DAB substrate kit (Vector). Sections were then mounted on slides, counterstained with hematoxylin, and inspected using a microscope (Nikon Eclipse 50i, Kodak Scientific Imaging System, New Haven, CT). Digital images were acquired using a camera (SPOT 7.4 Slider RTKE, Diagnostic Instruments, Sterling Heights, MI) connected to a computer. The anatomical structure of tissue sections were identified cytoarchitecturally using The Rat Brain Stereotaxic Coordinates[20]. Primary regions of interest included the central projections of the vagus nerve including the nucleus tractus solitarius, the locus coeruleus, and the cerebral cortex. Number of c-Fos positive cells in the nuclei tractus solitarii and the loci coerulei were counted from three consecutive sections and averaged for each animal blindly to the treatment groups, as published before [19].

Sample size and data analysis-We estimated that $\mathrm{n}=14$ in each group needed to detect $25 \%$ difference in infarct size at $\alpha=0.05$ and power of $90 \%$. The exclusion criteria were $<60 \%$ decrease in baseline laser Doppler signal upon MCAO, intracranial bleeding and infarction limited to the striatum. Continuous data were expressed as mean $\pm \mathrm{SD}$. Noncontinuous data were expressed as median \pm interquartile range (IQR). ABP, HR, arterial blood gases and $\mathrm{pH}$, and temperature were analyzed by repeated measures ANOVA, and when needed, by the Student-Newman-Keuls test. Infarct volumes were compared using an unpaired t-test. Neurological scores were compared using repeated measures ANOVA, and when needed, by the Mann-Whitney U test. Cell counts were analyzed using one-way ANOVA, and when needed, by the Student-Newman-Keuls test. Differences with $p<0.05$ were considered statistically significant.

\section{Results}

\section{1- The effect of aVNS on infarct size}

Three animals were excluded and replaced because MCAO did not induce $\geq 60 \%$ decrease in baseline rCBF. One animal in the treatment and two animals in the control group that died prematurely were replaced with additional animals to achieve the calculated sample size. Our mortality rate of $10 \%$ was comparable to the reported rates in other studies using the same model [21-23]. There was no difference in body temperature, brain temperature, and arterial blood gases and $\mathrm{pH}$ between control and treatment groups. There was no effect of aVNS on blood pressure and heart rate (Table 1). MCAO caused 68\% decrease in laser Doppler signal on average and this was fully reversed by reperfusion (Fig. 2A). aVNS 
slightly increased rCBF starting 50 min after the onset of stimulation but this effect did not reach a statistical significance (repeated measures ANOVA: $F(1,26)=3.313, p=0.080$ ). There were no concurrent changes in ABP (Fig. 2B; repeated measures of ANOVA: F(1,26) $=0.182, \mathrm{p}=0.673$ ) or HR (data not shown; repeated measures of ANOVA: $\mathrm{F}(1,26)=0.218$, $\mathrm{p}=0.644)$.

Transient occlusion of the right MCA resulted in a visible infarct in the cerebral cortex and the underlying striatum in all animals (Fig. 3A-B). The mean indirect infarct volume and SD was $44.20 \pm 7.58 \%$ in the control animals and $31.65 \pm 9.67 \%$ in aVNS-treated animals (unpaired t-test: $\mathrm{t}=2.059, \mathrm{p}<0.0001 ;$ Fig. $3 \mathrm{C}$ ). The mean direct infarct volume and SD was $47.00 \pm 8.62 \%$ in the control animals and $33.14 \pm 13.04 \%$ in aVNS-treated animals (unpaired t-test: $\mathrm{t}=3.317, \mathrm{p}<0.005$ ). aVNS-treated animals had better neurological scores 24 hours after ischemia compared to control animals (Fig. 3D). The median neurological score was $3.0 \pm 0.5$ at 3 hours and $3.0 \pm 0.0$ at 24 hours after ischemia in the control group. The corresponding scores in aVNS-treated animals were $3.0 \pm 0.5$ and $2.0 \pm 0.5$, respectively. The difference was significant at 24 hours (repeated measures ANOVA: $F(1,26)=43.105$, $\mathrm{p}<0.0001$; Mann-Whitney U test: $\mathrm{p}<0.0001)$.

\section{2- Brain regions activated by aVNS}

In all animals (sham, aVNS, cVNS, and trigeminal nerve stimulation), c-Fos staining was positive in the cingulate (Fig. 4A), fronto-parietal, and piriform cortices ipsilateral to the MCAO (not shown). Stimulation of the vagus nerve dermatome in the external ear resulted in bilateral c-Fos staining in the nuclei tractus solitarii (Fig. 4B) and loci coerulei in all animals. Additionally, there was c-Fos staining in the facial nucleus and the lateral reticular nucleus in one animal each. In cVNS-treated animals, there was stronger c-Fos staining in the nuclei tractus solitarii (Fig. 4C) and loci coerulei as compared to aVNS but this difference was not statistically significant. In contrast, trigeminal nerve stimulation did not result in c-Fos staining in the nucleus tractus solitarius (Fig. 4D) or locus coeruleus. The number of c-Fos positive cells in the ipsilateral and contralateral nuclei tractus solitarii were as follows, respectively: aVNS group $24.7 \pm 16.7$ and $20.2 \pm 8.2$; cVNS group $33.5 \pm 8.2$ and $31.5 \pm 6.1$; control group $0.8 \pm 1.0$ and $0.9 \pm 0.7$; trigeminal nerve stimulation group 2.0 \pm 0.8 and $0.5 \pm 0.6$. The number of c-Fos positive cells in the ipsilateral and contralateral loci coerulei were as follows, respectively: aVNS group $10.0 \pm 4.5$ and $8.7 \pm 4.9$; cVNS group $18.7 \pm 7.4$ and $16.5 \pm 9.1$; control group $1.2 \pm 1.2$ and $1.0 \pm 2.0$; trigeminal nerve stimulation group $2.4 \pm 0.8$ and 1.4 \pm 1.2 . The number of c-Fos positive cells in aVNS and cVNS groups were significantly different from control and trigeminal nerve stimulation groups in both nuclei tractus solitarii and loci coerulei ( $p<0.05$ vs. control and trigeminal nerve stimulation groups).

\section{Discussion}

Electrical stimulation of afferent vagus nerve fibers in the neck is a safe, FDA-approved treatment for refractory partial onset seizures and treatment-resistant depression. We have previously shown that direct stimulation of the vagus nerve in the neck reduces infarct volume by about $50 \%$ in rats [1]. However, the exact mechanism underlying cVNS-induced 
neuroprotection in acute cerebral ischemia is not known. It has been suggested that cVNS modulates cerebral blood flow, decreases neuronal excitability, and inhibits inflammation in animal models of epilepsy, traumatic brain injury as well as in healthy animals [24-26]. In this report, we build on the prior evidence by showing that a brief period of transcutaneous stimulation corresponding to the dermatome of the vagus nerve in the external ear initiated 30 min after contralateral transient MCAO reduces infarct volume by $28 \%$ in rats and leads to an improvement in neurological outcome that is sustained at 24 hours. We also show that afferent impulses from the external ear can reach to the brainstem and activate the nuclei (nucleus tractus solitarius and locus coeruleus) that mediate antiepileptic and antidepressive effects of cVNS [19,27], suggesting that aVNS-induced ischemic protection shares similar central patways to that of the gold standard cVNS.

The vagus nerve primarily provides parasympathetic innervation to the viscera. The only somatic sensory innervation for any branch of the vagus nerve is the external ear. Afferent nerve fibers from the cymba concha, cavum concha, antihelix, posterior aspect of the external auditory meatus, and the back of the ear travel with the auricular branch of the vagus nerve, reach the superior ganglion, and then terminate in the spinal trigeminal nucleus and to a lesser extent in the nucleus tractus solitarius [4,28-30]. In contrast to somatic sensory fibers, visceral sensory fibers (fibers that are typically activated during cVNS) synapse in the inferior ganglion, and their central processes enter the medulla to terminate mostly in the nucleus tractus solitarius and the dorsal motor nucleus of vagus, and, to a lesser extent, in the spinal trigeminal nucleus [31]. The nucleus tractus solitarius is the key relay nucleus that receives afferent vagal information and projects it to several subcortical structures including the locus coeruleus [32] and the thalamus [33], which, in turn, project to the cerebral cortex [34]. The present study shows that electrical stimulation of the vagus nerve dermatome in the external ear activates the central vagal pathway that projects from the nucleus tractus solitarius. This finding is in line with prior reports showing that aVNS causes reproducible vagus sensory evoked potentials from scalp and brain activation patterns in fMRI similar to cVNS activation in the nucleus tractus solitarius by aVNS in healthy volunteers $[5,6]$.

c-Fos is a commonly used surrogate marker for neuronal activation [35]. Under quiescent conditions there is little or no c-Fos expression in the brain [36]. Focal cerebral ischemia is a strong activator of c-Fos expression in areas surrounding the ischemia [37]. Accordingly, in this study we showed c-Fos staining in the ipsilateral fronto-parietal, cingulate, and piriform cortices after MCA occlusion. In addition, we also showed c-Fos staining in the nuclei tractus solitarii and loci coerulei in ischemic animals after aVNS and cVNS but not after trigeminal nerve stimulation. This is in line with prior studies of cVNS in healthy animals reporting c-Fos staining bilateraly in the nuclei tractus solitarii and loci coerulei, but not in the cerebral cortices [19]. These findings support the conclusion that aVNS is able to specifically activate those brain regions that are activated by cVNS.

Although aVNS caused significant reduction in infarct volume, the effect size was smaller than that reported for cVNS ( $28 \%$ vs. $50 \%$ ). This could be, in part, due to weaker activation of central vagal pathways by aVNS as compared to cVNS, as suggested by less intense cFos staining in the nuclei tractus solitarii of aVNS-treated animals. It is also possible that 
individual differences in sensory innervation of the cavum concha have contributed to the observed variance in effect size. Even though cavum concha has been reported to be the most efficient site in rat ear to induce autonomic nervous system changes[38], the innervation of the external ear shows significant variability [28]. We found that, in contrast to stimulation of the vagus nerve dermatome, stimulation of the dermatome innervated by the trigeminal nerve did not lead to typical activation pattern (i.e., c-Fos staining) in the nucleus tractus solitarius and locus coeruleus. Trigeminal nerve innervates the vast majority of the external ear including the regions that immediately surround the vagus nerve dermatome [28,30]. This highlights the importance of precise localization of the vagus nerve dermatome for a consistent response to electrical stimulation.

The present study has some limitations. We tested the effect of aVNS in healthy rats, in a single experimental model, and only up to 24 hours after ischemia. Studies using animals with comorbid conditions, utilizing other experimental stroke models (including but not limited to thromboembolic occlusion and distal MCA occlusion), and testing beyond 24 hours are warranted to evaluate the clinical potential of aVNS in stroke. Also, we used the same stimulation parameters used for cVNS in this study. Optimal stimulation parameters for aVNS may differ from those for cVNS. Future studies could maximize the therapeutic effect by varying stimulation parameters. In addition, it is important to note that although the expression of c-Fos is a valuable marker of neuronal activation, it may underestimate the effect as some neurons fail to express the gene or may not express it at detectable levels [39]. Alternative activation markers and combined use of stereological methods could better elucidate the neuroanatomic changes elicited by aVNS.

\section{Conclusions}

Although a number of neuroprotective pharmaceuticals with extensive theoretical appeal for the treatment of acute stroke have been developed, the vast majority of exogenously administered treatments or interventions tested to date for stroke have been either unfeasible, ineffective, or associated with harm. Such exogenous compounds attempt to circumvent or override the intrinsic neuroprotective system of the brain. Their ability to reach desired concentrations within the ischemic tissue is limited. Here, we describe a novel approach to leverage the brain's own intrinsic systems that render the brain more tolerant to ischemia. The significance of our finding is that, if verified in different experimental settings (i.e., different ischemia models, extended time frames, animals with comorbid conditions), it has potential to facilitate the development of innovative treatment paradigms focusing on external stimulation of the nervous system structures that diffusely connect to vascular and/or neural tissue and provide neuroprotection. If proven safe and effective, such methods could avoid missed opportunities to reduce ischemic brain injury as they can be easily applied as an adjunctive treatment, even in the ambulance.

\section{Acknowledgments}

\section{Sources of Funding}

This study was supported by American Heart Association (10SDG2600218 to I.A.). I.A. was also supported by NINDS (R21-NS081395). H.A. was supported by NINDS (RO1-NS059710), while V.N. was supported by 
NCCAM (P01-AT002048, R01-AT007550) and NIDDK (R21-DK097499). This research was carried out in whole at the Athinoula A. Martinos Center for Biomedical Imaging at the Massachusetts General Hospital, using resources provided by the Center for Functional Neuroimaging Technologies, P41RR14075, a P41 Regional Resource supported by the Biomedical Technology Program of the National Center for Research Resources (NCRR), National Institutes of Health.

\section{Abbreviations}

$\begin{array}{ll}\text { MCAO } & \text { Middle cerebral artery occlusion } \\ \text { cVNS } & \text { cervical vagus nerve stimulation } \\ \text { aVNS } & \text { auricular vagus nerve stimulation } \\ \text { ABP } & \text { arterial blood pressure } \\ \text { HR } & \text { heart rate } \\ \text { rCBF } & \text { regional cerebral blood flow } \\ \text { TTC } & \text { 2,3,5-triphenyltetrazolium chloride }\end{array}$

\section{References}

1. Ay I, Lu J, Ay H, Gregory Sorensen A. Vagus nerve stimulation reduces infarct size in rat focal cerebral ischemia. Neurosci Lett. 2009; 459:147-151. [PubMed: 19446004]

2. Ay I, Sorensen AG, Ay H. Vagus nerve stimulation reduces infarct size in rat focal cerebral ischemia: An unlikely role for cerebral blood flow. Brain Res. 2011; 1392:110-115. [PubMed: 21458427]

3. Hiraki T, Baker W, Greenberg JH. Effect of vagus nerve stimulation during transient focal cerebral ischemia on chronic outcome in rats. J Neurosci Res. 2012; 90:887-894. [PubMed: 22420043]

4. Kiernan, JA. Barr's The Human Nervous System: An Anatomical Viewpoint. 9. Baltimore: Lippincott Williams \& Wilkins; 2009. p. 134

5. Fallgatter AJ, Neuhauser B, Herrmann MJ, Ehlis AC, Wagener A, Scheuerpflug P, Reiners K, Riederer P. Far field potentials from the brain stem after transcutaneous vagus nerve stimulation. J Neural Transm. 2003; 110:1437-1443. [PubMed: 14666414]

6. Kraus T, Hosl K, Kiess O, Schanze A, Kornhuber J, Forster C. Bold fmri deactivation of limbic and temporal brain structures and mood enhancing effect by transcutaneous vagus nerve stimulation. J Neural Transm. 2007; 114:1485-1493. [PubMed: 17564758]

7. Fallgatter AJ, Ehlis AC, Ringel TM, Herrmann MJ. Age effect on far field potentials from the brain stem after transcutaneous vagus nerve stimulation. Int J Psychophysiol. 2005; 56:37-43. [PubMed: 15725488]

8. Polak T, Markulin F, Ehlis AC, Langer JB, Ringel TM, Fallgatter AJ. Far field potentials from brain stem after transcutaneous vagus nerve stimulation: Optimization of stimulation and recording parameters. J Neural Transm. 2009; 116:1237-1242. [PubMed: 19728032]

9. He W, Jing XH, Zhu B, Zhu XL, Li L, Bai WZ, Ben H. The auriculo-vagal afferent pathway and its role in seizure suppression in rats. BMC Neurosci. 2013; 14:85. [PubMed: 23927528]

10. Folan-Curran J, Hickey K, Monkhouse WS. Innervation of the rat external auditory meatus: A retrograde tracing study. Somatosens Mot Res. 1994; 11:65-68. [PubMed: 8017145]

11. Napadow V, Edwards RR, Cahalan CM, Mensing G, Greenbaum S, Valovska A, Li A, Kim J, Maeda Y, Park K, Wasan AD. Evoked pain analgesia in chronic pelvic pain patients using respiratory-gated auricular vagal afferent nerve stimulation. Pain Med. 2012; 13:777-789. [PubMed: 22568773]

12. Busch V, Zeman F, Heckel A, Menne F, Ellrich J, Eichhammer P. The effect of transcutaneous vagus nerve stimulation on pain perception - an experimental study. Brain Stimul. 2013; 6:202209. [PubMed: 22621941] 
13. Ay I, Ay H. Ablation of the sphenopalatine ganglion does not attenuate the infarct reducing effect of vagus nerve stimulation. Auton Neurosci. 2013; 174:31-35. [PubMed: 23273773]

14. Schmid-Elsaesser R, Zausinger S, Hungerhuber E, Baethmann A, Reulen HJ. A critical reevaluation of the intraluminal thread model of focal cerebral ischemia: Evidence of inadvertent premature reperfusion and subarachnoid hemorrhage in rats by laser-doppler flowmetry. Stroke. 1998; 29:2162-2170. [PubMed: 9756599]

15. Lumenta DB, Plesnila N, Klasner B, Baethmann A, Pruneau D, Schmid-Elsaesser R, Zausinger S. Neuroprotective effects of a postischemic treatment with a bradykinin b2 receptor antagonist in a rat model of temporary focal cerebral ischemia. Brain Res. 2006; 1069:227-234. [PubMed: 16378603]

16. Longa EZ, Weinstein PR, Carlson S, Cummins R. Reversible middle cerebral artery occlusion without craniectomy in rats. Stroke. 1989; 20:84-91. [PubMed: 2643202]

17. Smith DC, Modglin AA, Roosevelt RW, Neese SL, Jensen RA, Browning RA, Clough RW. Electrical stimulation of the vagus nerve enhances cognitive and motor recovery following moderate fluid percussion injury in the rat. J Neurotrauma. 2005; 22:1485-1502. [PubMed: 16379585]

18. Bederson JB, Pitts LH, Tsuji M, Nishimura MC, Davis RL, Bartkowski H. Rat middle cerebral artery occlusion: Evaluation of the model and development of a neurologic examination. Stroke. 1986; 17:472-476. [PubMed: 3715945]

19. Cunningham JT, Mifflin SW, Gould GG, Frazer A. Induction of c-fos and deltafosb immunoreactivity in rat brain by vagal nerve stimulation. Neuropsychopharmacology. 2008; 33:1884-1895. [PubMed: 17957222]

20. Paxinos, G.; Watson, C. The Rat Brain in Stereotaxic Coordinates. 6. San Diego: Academic Press; 2007.

21. Mokudai T, Ayoub IA, Sakakibara Y, Lee EJ, Ogilvy CS, Maynard KI. Delayed treatment with nicotinamide (vitamin $\mathrm{b}(3)$ ) improves neurological outcome and reduces infarct volume after transient focal cerebral ischemia in wistar rats. Stroke. 2000; 31:1679-1685. [PubMed: 10884473]

22. Knight RA, Barker PB, Fagan SC, Li Y, Jacobs MA, Welch KM. Prediction of impending hemorrhagic transformation in ischemic stroke using magnetic resonance imaging in rats. Stroke. 1998; 29:144-151. [PubMed: 9445344]

23. Popp A, Jaenisch N, Witte OW, Frahm C. Identification of ischemic regions in a rat model of stroke. PLoS One. 2009; 4:e4764. [PubMed: 19274095]

24. Martle V, Peremans K, Raedt R, Vermeire S, Vonck K, Boon P, Van Ham L, Tshamala M, Caemaert J, Dobbeleir A, Duchateau L, Waelbers T, Gielen I, Bhatti S. Regional brain perfusion changes during standard and microburst vagus nerve stimulation in dogs. Epilepsy Res. 2014:S0920-1211. [PubMed: 24630046]

25. Mollet L, Grimonprez A, Raedt R, Delbeke J, El Tahry R, De Herdt V, Meurs A, Wadman W, Boon P, Vonck K. Intensity-dependent modulatory effects of vagus nerve stimulation on cortical excitability. Acta Neurol Scand. 2013; 128:391-396. [PubMed: 23614853]

26. Bansal V, Ryu SY, Lopez N, Allexan S, Krzyzaniak M, Eliceiri B, Baird A, Coimbra R. Vagal stimulation modulates inflammation through a ghrelin mediated mechanism in traumatic brain injury. Inflammation. 2012; 35:214-220. [PubMed: 21360048]

27. Krahl SE, Clark KB, Smith DC, Browning RA. Locus coeruleus lesions suppress the seizureattenuating effects of vagus nerve stimulation. Epilepsia. 1998; 39:709-714. [PubMed: 9670898]

28. Peuker ET, Filler TJ. The nerve supply of the human auricle. Clin Anat. 2002; 15:35-37. [PubMed: 11835542]

29. Nomura S, Mizuno N. Central distribution of primary afferent fibers in the arnold's nerve (the auricular branch of the vagus nerve): A transganglionic hrp study in the cat. Brain Res. 1984; 292:199-205. [PubMed: 6692153]

30. Gray, H. Anatomy of the Human Body. 20. Vol. chapter 5j. Philadelphia: Lea \& Febiger; 2000.

31. Nomura S, Mizuno N. Central distribution of efferent and afferent components of the cervical branches of the vagus nerve. A hrp study in the cat. Anat Embryol (Berl). 1983; 166:1-18. [PubMed: 6837927] 
32. Cedarbaum JM, Aghajanian GK. Afferent projections to the rat locus coeruleus as determined by a retrograde tracing technique. J Comp Neurol. 1978; 178:1-16. [PubMed: 632368]

33. Ricardo JA, Koh ET. Anatomical evidence of direct projections from the nucleus of the solitary tract to the hypothalamus, amygdala, and other forebrain structures in the rat. Brain Res. 1978; 153:1-26. [PubMed: 679038]

34. Henry TR. Therapeutic mechanisms of vagus nerve stimulation. Neurology. 2002; 59:S3-14. [PubMed: 12270962]

35. Kovacs KJ. C-fos as a transcription factor: A stressful (re)view from a functional map. Neurochem Int. 1998; 33:287-297. [PubMed: 9840219]

36. Dragunow M, Faull R. The use of c-fos as a metabolic marker in neuronal pathway tracing. J Neurosci Methods. 1989; 29:261-265. [PubMed: 2507830]

37. Uemura Y, Kowall NW, Moskowitz MA. Focal ischemia in rats causes time-dependent expression of c-fos protein immunoreactivity in widespread regions of ipsilateral cortex. Brain Res. 1991; 552:99-105. [PubMed: 1913186]

38. Gao XY, Zhang SP, Zhu B, Zhang HQ. Investigation of specificity of auricular acupuncture points in regulation of autonomic function in anesthetized rats. Auton Neurosci. 2008; 138:50-56. [PubMed: 18068545]

39. Osharina V, Bagaev V, Wallois F, Larnicol N. Autonomic response and fos expression in the nts following intermittent vagal stimulation: Importance of pulse frequency. Auton Neurosci. 2006; 126-127:72-80. 
- aVNS decreases tissue damage after ischemic brain injury.

- aVNS increases neurological outcome after ischemic brain injury.

- $\quad$ aVNS induces in bilateral c-Fos staining in the nuclei tractus solitarii.

- aVNS induces in bilateral c-Fos staining in the loci coerulei.

- aVNS has no effect on arterial blood pressure and heart rate. 


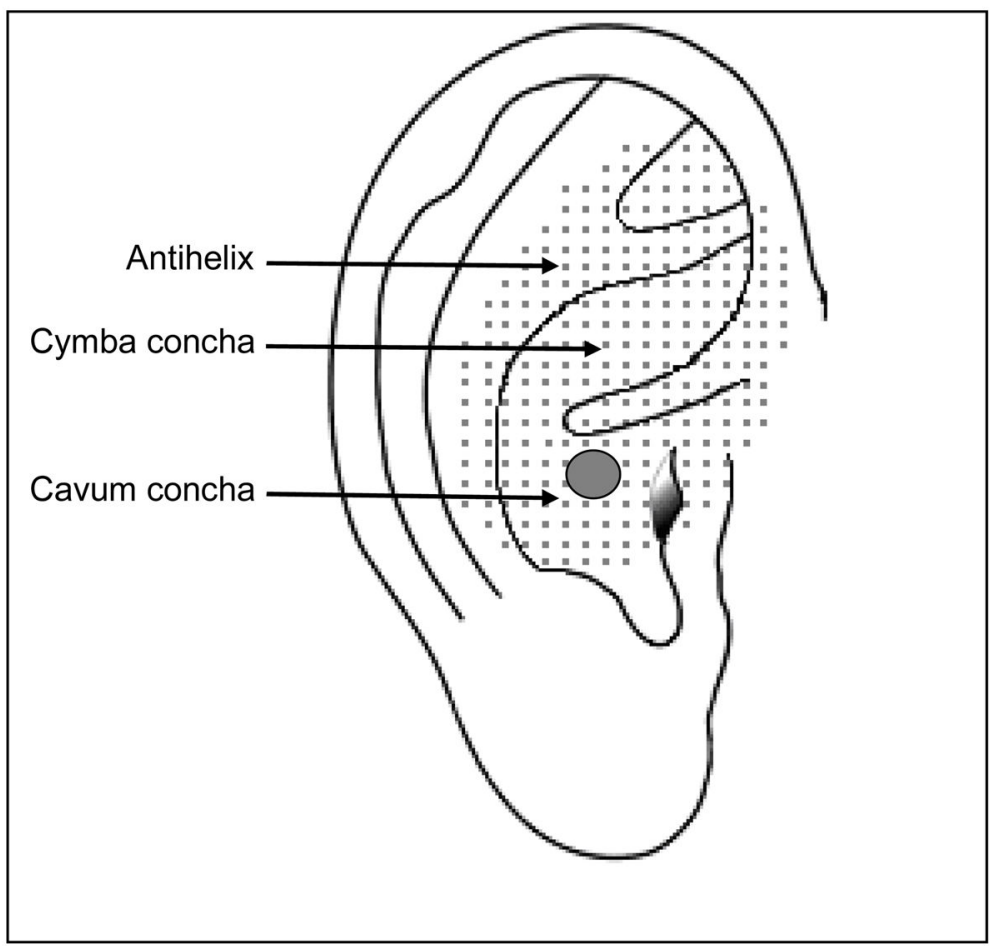

Figure 1.

Schematic representation of the ear. Shaded area shows the dermatome corresponding to the auricular branch of the vagus nerve. Circle illustrates the electrode implantation site. 
A

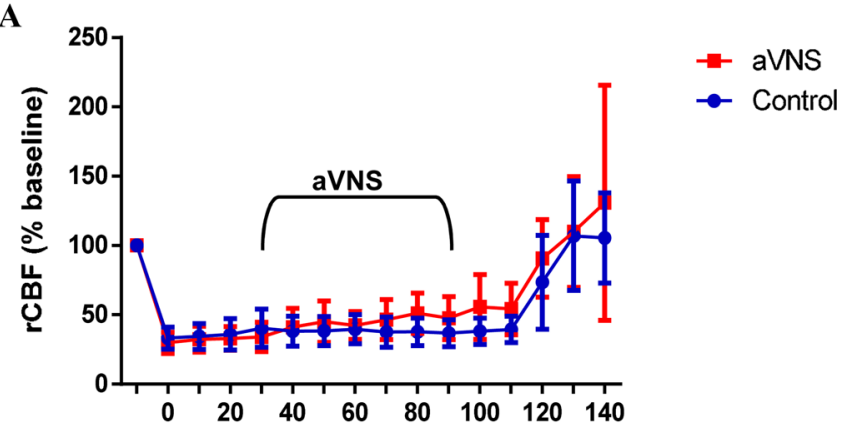

Time (min after MCAO)

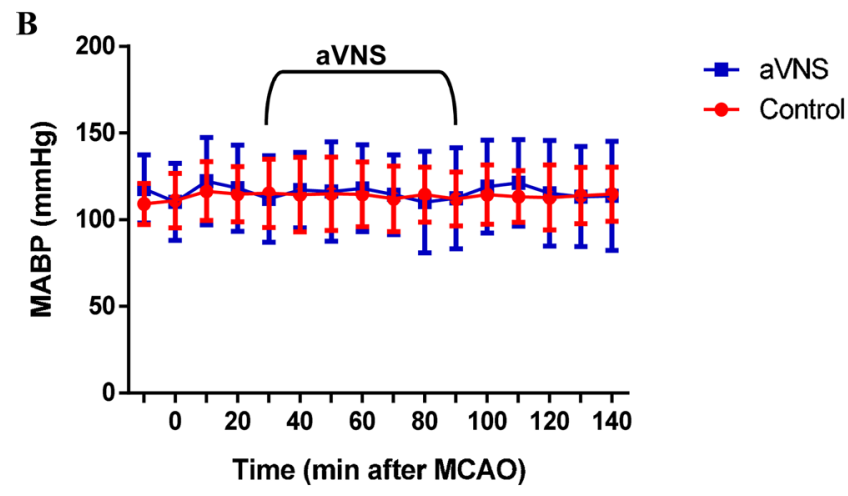

Figure 2.

aVNS has no statistically significant effect on physiological parameters. However, there was a trend toward an increase in $\mathrm{rCBF}(\mathrm{A})$ without any observable effect on mean $\mathrm{ABP}$ $(\mathrm{MABP})(\mathrm{B})$ during the experimental period. 

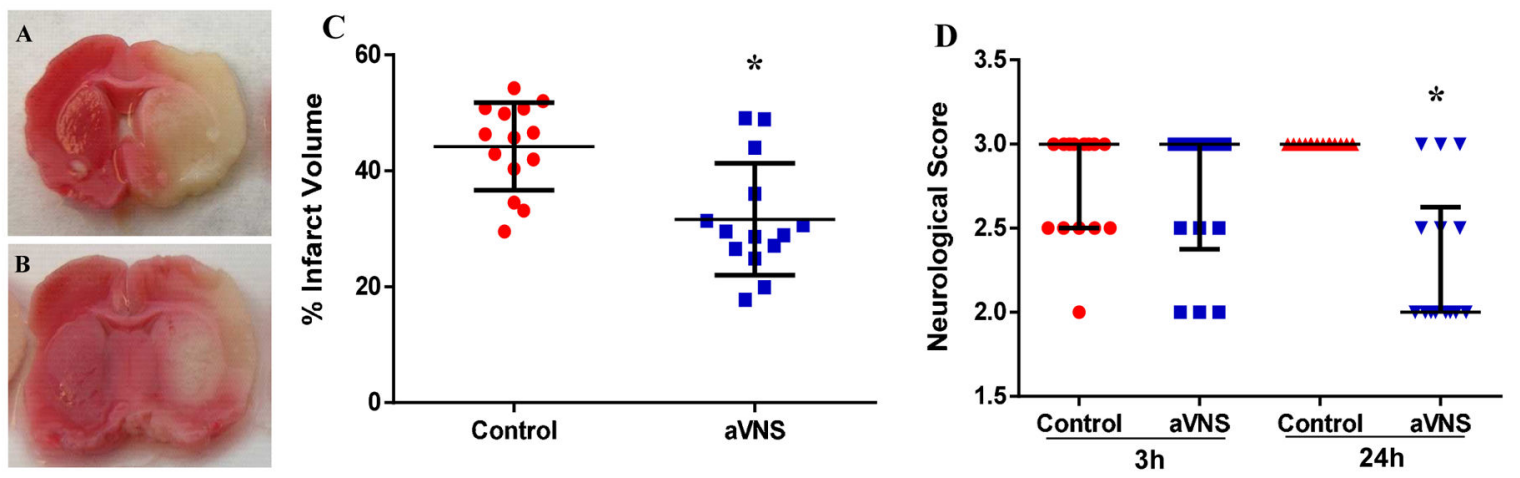

Figure 3.

aVNS has therapeutic effect in transient MCAO. Representative images of TTC-stained sections at the level of bregma $0.4 \mathrm{~mm}$ in control (A) and aVNS-treated (B) animals show comparably smaller infarct area in treated animals. Graphs demonstrate that infarct size (C) was smaller and neurological score (D) was better after aVNS treatment compared to control animals. ${ }^{*} \mathrm{p}<0.05$ vs. control. 

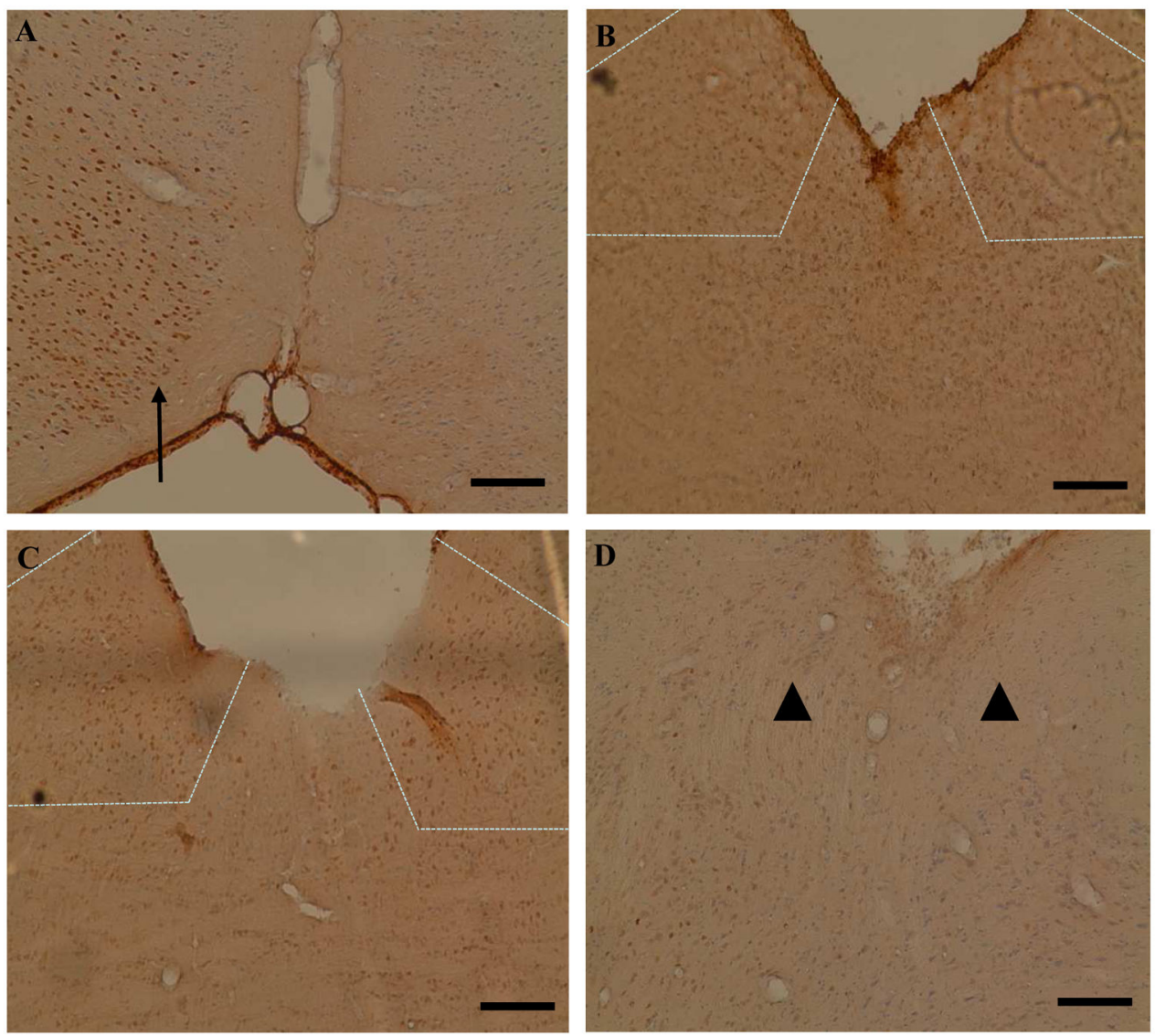

Figure 4.

aVNS activates vagal brainstem centers. Representative c-Fos immunohistochemistry images from cerebral cortex (A) and brainstem (B-D). In all animals, ischemia caused a positive immunostaining in the ipsilateral cingulate cortex (arrow in A) and the piriform cortex (not shown). In the fronto-parietal cortex, regions that correspond to penumbra had positive staining whereas regions corresponding to the core had no staining in all animals (not shown). Both aVNS (B) and cVNS (C)-treatment resulted in c-Fos staining in the ipsilateral and contralateral nuclei tractus solitarii (delineated by dashed lines). Stimulation of the trigeminal nerve dermatome resulted in no detectable staining in the nucleus tractus solitarius (arrowheads in D). Scale bar: $250 \mu \mathrm{m}$ in A; $100 \mu \mathrm{m}$ in B, C, D. 


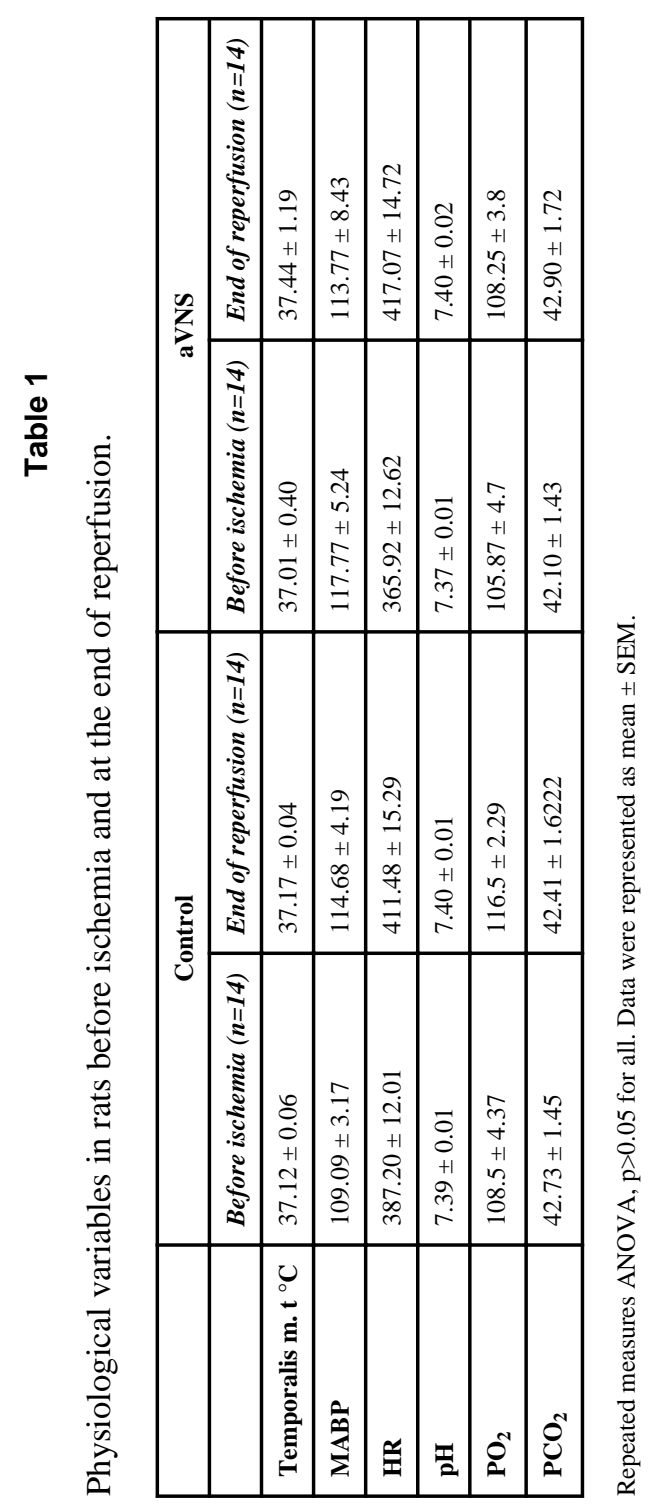

Brain Stimul. Author manuscript; available in PMC 2016 January 01. 